\title{
Outcome of Laparoscopic Ovarian Drilling (LOD) for Women with Polycystic Ovary Syndrome (PCOS): A retrospective case note review
}

\author{
Rifat Syed', Jamal Zaidi², Nabeha Dhar ${ }^{3}$ \\ 'Darent Valley Hospital, Darenth Wood Rd, Dartford DA2 8DA, UK \\ ${ }^{2}$ Conquest Hospital, The Ridge, Hastings, Saint Leonards-on-sea TN37 7RD, UK \\ ${ }^{3} 59$ Tonbridge Road, Hildenborough TN11 9BH, UK
}

Corresponding Author

Rifat Syed, Darenth Wood Rd, Dartford DA2 8DA, e-mail: syed.rifat@yahoo.co.uk

\section{Abstract}

Objective: To evaluate the effectivity of laparoscopic ovarian drilling procedures at the East Sussex Healthcare Trust, UK, over the past decade on sub-fertile women with polycystic ovary syndrome, regardless of clomiphene resistance.

Design: Retrospective case note review of LOD procedures

Methods: Study 1: Evaluating a systematic literature review investigating ovulation and pregnancy rates following LOD. Study 2: An audit of 58 women with LOD treated for ovulation induction at the ESHT fertility clinic between 2005-2014.

Main outcome measures: Ovulation, pregnancy, live birth, miscarriage rates; tubal patency; associated pathologies; previous treatments compared with Cochrane Review 2012.

Results: Study 1: From the literature review, 71\% achieved ovulation, whereas the pregnancy, live birth and miscarriage rates were $25-51 \%, 24-44 \%$ and $4-9 \%$ respectively. Study 2: Of the 58 patients studied, ovulation rate was $69 \%$, pregnancy rate was $39.4 \%$ and live birth rate was $30.3 \%$. The miscarriage rate was $15.4 \%$. Pregnancy outcome after LOD, irrespective of further treatment, showed 34 pregnancies. Including 17 spontaneous pregnancies and 17 pregnancies after further treatment. Of the 34 pregnancies, $85.3 \%$ were live birth, $11.7 \%$ miscarriages and 1 ectopic pregnancy.

Conclusion: No significant difference in ovulation rates post-LOD in ESHT, which is comparable to published studies. The low pregnancy and live birth rates indicate that additional factors affect the success of LOD. Co-existing pathology is associated with lower pregnancy rate post-LOD. Novel theories are postulated to explain underlying pathologies, thus PCOS treatment may change and open a fascinating area of research.

Key words: Polycystic ovary syndrome, laparoscopic ovarian drilling, clomiphene citrate 


\section{Introduction}

The key of the survival of humans is a functioning and intact reproductive system. Disorders in this system cause many problems; they lead to psychological, medical and economic complications. Infertility is the main cause of sadness, distress and isolation. Recent research has found that infertility has a severe impact on relationships. Current statistics have shown that one in seven couples are infertile in the UK. As medical science and technology have advanced it has been possible to identify most of the root causes of infertility in both women and men, however, a minority of the causes remain undiagnosed as being idiopathic or unexplained. A small number of patients have genetic disorders ${ }^{1}$. Genetic disorders of infertility have often been due to hormonal imbalance or digenetic gonads. After the cause of infertility has been found the aim is to correct all reversible aetiologies and overcome irreversible factors.

Literature reviews have described various genes which play a role in the development and regulation of the hypothalamic-pituitary-gonadal (HPG) axis.

PCOS is one of the commonest endocrinopathy affecting up to $20 \%$ of women. It is a common hormonal imbalance in women due to a combination of genetic and environmental factors, presenting with a variety of menstrual cycle disturbances. PCOS accounts for 90 $95 \%$ of women with anovulation who attend fertility clinic. This disorder affects a fifth of women during their reproductive age ${ }^{2}$. PCOS also called hyperandrogenic anovulation (HA) or Stein-Leventhal syndrome was first diagnosed by Stein and Levanthal in 1935.

It is diagnosed by Rotterdam criteria established by the European Society of Human Reproduction and Embryology in 2003; two out of the three criteria are required to confirm PCOS:

- Oligo-ovulation or anovulation

- Clinical and/or biochemical signs of hyper androgenization

- Ultrasonographic evidence of polycystic ovaries.
PCO is a condition where the ovaries have many cysts in ultrasound but do not have any symptoms or clinical changes of PCOS.

\section{Pathogenesis}

The familial pattern suggests genetic component and current literature suggests that PCOS in families resembles an autosomal dominant pattern ${ }^{3}$. The evidence for chronobiological abnormality in secretion of luteinizing hormone is not clear, whether the abnormality is in the hypothalamus or pituitary gland $^{4}$. In PCOS, ovaries produce excessive amounts of male hormones (androgen) by either the release of excessive luteinizing hormone ( $\mathrm{LH}$ ) by the anterior pituitary, or through high levels of insulin in the blood (hyperinsulinemia). Increased ovarian androgens are due to defects in the androgen synthesis. Elevated SRD5A activity in PCO showed that 5 alpha-reductase activity (SRD5A) may play a role in the pathogenesis of PCOS. The familial aggregation of hyperandrogenaemia in PCOS suggests that it is a genetic trait ${ }^{5}$. Prevalence of hypothyroidism is high in PCO patients and all patients should be screened. The androgen levels must be measured at around 8 am, the peak time of androgen production (PCOS in paediatric population) ${ }^{6}$, see table 1 .

\section{Management}

There are several treatment options for infertility related to anovulation due to PCOS. Management options usually depend on the reason the patient is seeking treatment and are individually based. Many treatment options, medical and surgical, are available. PCOS with infertility is the commonest reason for seeking treatment. Obesity in PCOS is associated with failure or delayed response to $\mathrm{CC}$, gonadotrophins and LOD. So, weight loss is considered as a first line therapy in obese patient with PCOS.

In 2013 NICE guidelines about weight reduction and first line treatment for PCOS were established. 
Table 1. Summarization of various laboratory tests. The androgen levels must be measured at around $8 \mathrm{am}$, the peak time of androgen production. (PCOS in paediatric population) ${ }^{6}$.

\section{LAB OR IMAGING STUDY}

Free testosterone

Total testosterone

Sex hormone binding globulin

LH.FSH

Prolactin

TSH. Free T4

DHEAS 17 alpha-hydroxyprogesterone

Morning cortisol level and/or 24-hour urinary free cortisol

Beta-HCG

Consider insulin-like growth factor 1 (IGF-1)

Lipid profile

\section{EXPECTED RESULT OR EXPLANATION}

Elevated in PCOS.

Elevated in PCOS.

Suppressed in PCOS.

Generally, LH is higher than FSH: older criteria no however this is not always seen.

To exclude hyperprolactinemia

To exclude hypothyroidism.

To screen for $\mathrm{CAH}$, androgen-secreting tumours (in is significantly elevated, generally over $600-700 \mathrm{n}$.

To exclude Cushing syndrome.

To exclude pregnancy.

To exclude acromegaly

IGF-1, like insulin, can increase androgen production of sex hormone binding globin.

To screen for hyperlipidaemia.
Weight reduction helps to decrease androgen, $\mathrm{LH}$ and insulin levels. This helps in regulating ovulation and improves the outcome of pregnancy in $\mathrm{PCOS}^{7}$. The first line of treatment is with CC (a selective oestrogen receptor modulator or SERM), or a combination of clomiphene and metformin as per the NICE 2013. However, the Cochrane review in 2012 showed that metformin improves the number of pregnancies but has no benefit in live birth rates ${ }^{8}$. CC is the gold standard for ovulation induction; however multiple pregnancy and CC resistance are drawbacks. If there is no ovulation after $150 \mathrm{mg}$ CC given for 3 consecutive months or a total therapy of 6 months, clomiphene resistance is considered. LOD is an alternative to ovulation induction with gonadotrophins for CC resistant PCOS. As per ESHRE/ASRM LOD is the second line treatment for ovulation induction in patients with CC resistance. Women who are clomiphene resistant can be treated with gonadotrophins, but often have an overproduction of follicles and are exposed to the risks of OHSS and multiple pregnancies. Go- nadotrophins are an expensive and time-consuming form of treatment requiring intensive monitoring. A Cochrane review of six randomized trials compared LOD with gonadotrophins showed a similar rate of ovulation, pregnancy outcome and miscarriage, no case of ovarian hyper stimulation was seen in either'. In LOD, however, low costs and even lower rates of multiple gestations were recorded.

\section{Laparoscopic Ovarian Drilling}

In 1930 Stein Leventhal started bilateral ovarian wedge resection of the ovaries in patients with PCOS to restore regular periods and pregnancy. The treatment was abandoned due to postoperative complications like peri-ovarian adhesions and introduction of medical management by clomiphene citrate. There are many different techniques for laparoscopic procedure used for bilateral ovarian wedge resection in PCOS. These procedures include:

- Bevelling the ovary on its longitudinal axis

- Multiple biopsies along longitudinal axis 
- Dissecting multiple sub capsular cysts

- Making multiple holes in the ovary (drilling)

LOD was first introduced by Gjonnaess, 1984. The mechanism of action of LOD and ovarian wedge resection are similar. Both procedures destroy ovarian androgen-producing tissue and reduce the peripheral conversion of androgens to oestrogens. A fall in the serum levels of androgens and LH and an increase in FSH levels have been demonstrated after ovarian drilling ${ }^{10}$. LOD causes the destruction of ovarian stroma which reduces circulating levels of androgens, like DHEAS and testosterone ${ }^{11}$. LOD helps in restoring the hormonal environment to normal; it produces non-steroidal factors which restore hypothalamo-pituitary-ovarian feedback mechanism ${ }^{12}$. LOD increases the ovarian growth factor (IGF-I), which increases the FSH and several endocrine changes occur, these result in the restoration of ovulation ${ }^{13}$. LOD can be done by heat, monopolar and bipolar, or lasers, as well as electrocautery. Currently unipolar needle electrode is used for LOD and four-six punctures of each ovary produce fewer adhesion formations and thermal damage to the stroma of the ovary. The number of punctures is variable and range from 4-12 in each ovary depending on the surgical preference.

The outcome of LOD in achieving ovulation and pregnancy rate was studied by Gjonaess and Naether in 1994 and they found similar ovulation rates of $82 \%$ and $92 \%$; the pregnancy rates of $69 \%$ and $70 \%$ and an abortion rate of 15\%. LOD increases the sensitivity of gonadotrophins in PCOS. A study by Behery in 2011 showed that LOD reduces ovarian vascularisation and reduces serum level of vascular endothelial growth factor (VEGF). A recent Cochrane review found that LOD has a pregnancy rate of $39.7 \%$ and a live birth rate of $33.9 \%$, without an increased risk of multiple pregnancy and OHSS. Recent studies on comparison between unilateral and bilateral ovarian drilling showed that there is no difference in the outcomes of ovulation and pregnancy rate between the two methods ${ }^{14}$. Recent studies have shown that ovulation persists over a longer period in LOD and the procedure is performed only once, and no monitoring is needed.

\section{Method}

\section{Study 1}

What is the strength of evidence documenting the outcome of ovarian drilling for PCOS in sub fertility clinic? - A systematic review?

Using the search term "PCOS and management with LOD and reproductive outcome", I performed a secondary analysis of articles through a literature search using Medline, PubMed search EMBASE, World Health Organisation (WHO) and the Cochrane Central Register of Controlled Trials. 35 papers on the factors affecting LOD were reviewed. Additionally, the papers on Serum vascular factors in PCOS and its relation to $\mathrm{LOD}^{15}$ and the impact of preoperative FSH on the long-term outcome of LOD in women with clomiphene resistant $\mathrm{PCOS}^{16}$ were also reviewed. Recent research on the importance of LOD to be offered earlier to clomiphene resistant anovulatory women with PCOS was also reviewed.

20 papers were identified that referred to the use of LOD in PCOS patients using MEDLINE. The papers spanned the years $2005-2015$. They were then individually assessed for their value in the efficacy of the treatment with LOD in PCOS. Papers were excluded if the unilateral LOD was done in PCOS patients and if LOD was done to see the effect on circulating inhibin B. Papers were also excluded if the study group size was too small to be a fair test of efficiency.

\section{Study 2}

What is the success of the outcome of ovarian drilling for PCOS in a sub-fertility clinic?

I carried out retrospective audit for PCOS patients who underwent LOD. Audit standard was based on 
Cochrane database for LOD by diathermy or laser for ovulation induction in anovulatory PCOS. A list of patients was obtained from the Clinical Effectiveness Coordinator and a specific proforma was designed and used for this audit. A total of 80 patients who underwent LOD between 2005 and 2014 were identified and evaluated retrospectively. Notes were reviewed for the accuracy of diagnosis, 64 sets of notes were available for the audit, $80 \%$ of the total number of patients. For the purpose of this study, the inclusion criterion was the "Rotterdam criteria" in which menstrual cycle abnormalities and ultrasound findings were used to diagnose PCOS patients who underwent LOD. Six patients failed to be consistent with this, thus a total of 58/64 (72.5\%) case-notes were reviewed. Each set of available medical records were examined, and appropriate data entered on to a spread sheet. The patients who were excluded were those that had been treated with LOD for other purposes. Three cases were not performed on fertility patients and one case was found to have ovarian borderline tumour and was referred to the oncology team; attempt to conceive ceased. One case was wrongly coded as she has an ovarian cyst drained followed by TAH + BSO later. One case was not clearly documented in the operative notes whether she had the procedure or not. All 58 cases were infertile and required ovulation induction with the intervention to conceive. The 56 PCOS patients appeared to have oligomenorrhea and two had normal menstrual cycles. Regarding ultrasound findings, 56 patients had PCO scan picture and 2 cases had normal scan. Seminal analysis was evaluated in all 58 cases of PCOS and 50 cases had normal sperm counts, seven cases had mild male factor abnormality and for one case it was undocumented.

LOD was performed in 58 cases and an operative finding associated with other conditions was checked. Patients were divided into two diagnostic groups, PCOS associated with other pathologies found at LOD and patients found to have only PCOS "pure". There were
25/58 (43.1\%) patients who had PCOS associated with other pathologies and 33/58(56.8\%) patients with "pure" PCOS. The pregnancy rate was less in patients associated with other pathologies 4/25 (16\%) while the pregnancy rate in pure PCOS was $39.4 \%$.

\section{Statistical analysis}

The difference in the ovulation pregnancy and live birth between the two diagnostic groups of pure PCOS and in PCOS patients associated with other pathologies was calculated using the Chi-squared test. Mann Witney test was used to determine the statistically relevance of the pregnancy rate, miscarriage rate and live birth rate of the two groups. In addition, the difference between our LOD result with Cochrane review in PCOS patients with no other pathology was calculated using Chi-squared test. Statistical significance was identified as $\mathrm{p}<0.05$.

\section{Results}

\section{Study 1: Systematic review}

Eight studies have used LOD in PCOS along with the success rates. Table 2 shows the median ovulation rate per cycle was $79.41 \%$ (range $75-88 \%$ ) respectively. The median pregnancy rate was $49.20 \%$ (range 45-50\%) respectively. The total number of patients undergoing LOD according to these reports is 814 patients. This illustrates the effectiveness of LOD in inducing ovulation, but also highlights the other parameters involved with the success of the pregnancy.

\section{Study 2: Result of patients treated in ESH}

58 cases notes were reviewed. The demographic data age, BMI, parity and duration of sub fertility were looked at for every case. The BMI prior to LOD was calculated in addition to the age. Moreover, parity of the patient and duration of infertility were evaluated prior to LOD. In addition, medical treatment before LOD with CC and other medicine was evaluated. 
Table 2. Summary of existing literature using LOD in PCOS patients to date. The data includes the ovulation and pregnancy success rates.

$\begin{array}{lc}\text { AUTHORS } & \text { NO OF PATIENTS } \\ \text { Zakherah et al., 2010 } & 150 \\ \text { Hameed et al., 2011 } & 69 \\ \text { Radu et al., 2011 } & 107 \\ \text { Enzenwa et al., 2012 } & 54 \\ \text { Kaur et al., 2013 } & 100 \\ \text { Onofriescu et al., 2013 } & 40 \\ \text { Abuelghar et al., 2014 } & 251 \\ \text { Yanamandra et al., 2015 } & 43\end{array}$

\section{Age}

Most patients were 27-31 years old, 21 out of 58 (36\%). There were also 18 out of $58(31 \%)$ patients aged 20-26. Although, 19 (34\%) patients were more than 32 years as shown in figure 1.

\section{Parity}

45 patients were nulliparous (never pregnant) and 13 were multiparous (pregnant previously). In the multiparous women 2 patients had 1 miscarriage and 4 patients had 2 miscarriages, although the 13 multiparous had secondary infertility. 9 patients were para 1 and 3 patients were para 2 , whereas only 1 patient was para 3 as shown in table 3.

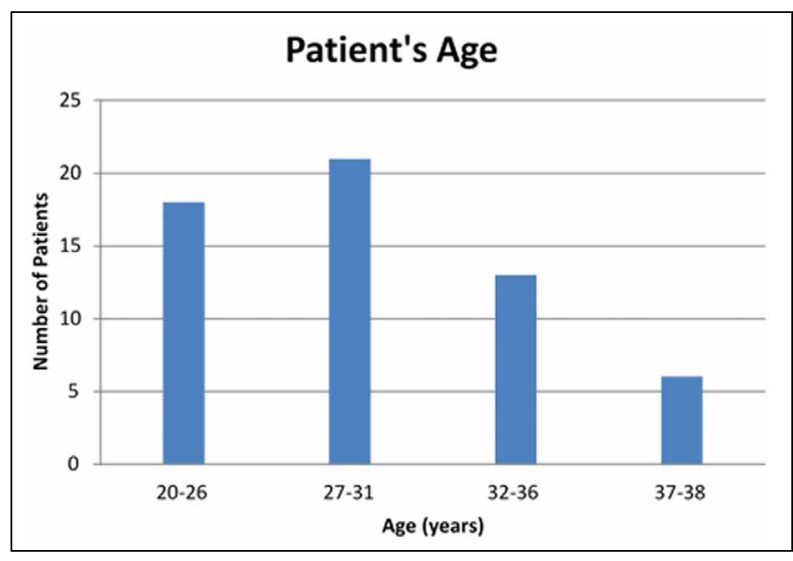

Figure 1. Age of the patients when undergoing LOD.

$\begin{array}{cc}\text { REGULAR CYCLES } & \text { PREGNANCY RATE } \\ 85.3 \% & 50.7 \% \\ 82.61 \% & 47.82 \% \\ 74 \% & 35 \% \\ 74.6 \% & 40.7 \% \\ 76.2 \% & 47.3 \% \\ 85 \% & 80 \% \\ 88.24 \% & 53.5 \% \\ 70 \% & 53.5 \%\end{array}$

\section{BMI}

Twenty patients were between BMI 20 to 25, twenty-one patients were between BMI $26-30$, nine patients were between BMI 31 to 35, two patients were BMI 36 to 40 , one patient was BMI 43, one was BMI 42 and BMI was not recorded for 4 patients. Median BMI was 20 to 30 as shown in figure 2 .

\section{Duration of sub fertility}

Thirteen patients were found to be sub fertile from one year, fourteen patients were sub fertile from 2 years, 13 patients were sub fertile from 3 to 4 years, 9 patients were sub fertile from 5 to 6 years, 2 patients were sub fertile from 7 years and

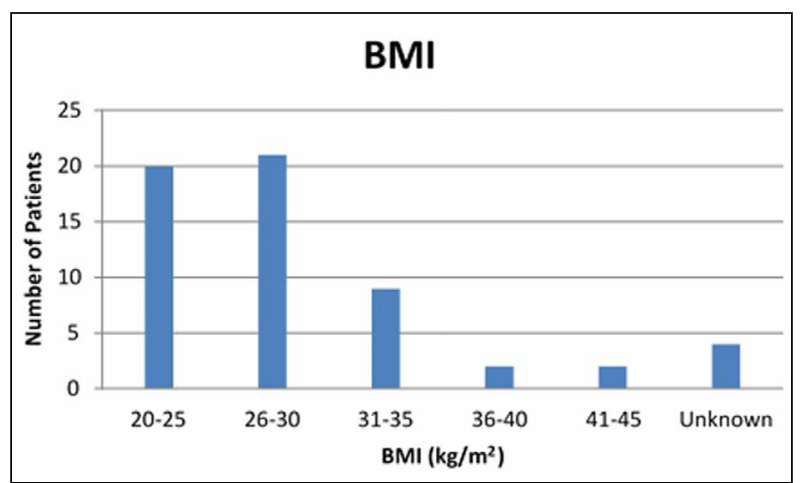

Figure 2. BMI of 58 patients when having LOD. 
Table 3. Parity (number of children) in 58 PCOS patients before LOD.

PARA 0

39
PARA 0+1

2
PARA 0+2

4
PARA 1
PARA 2

3
PARA 3

1 the sub fertility for 7 patients was unknown. However, majority of patients were sub fertile from 1- 4 years (see figure 3).

\section{Clinical features before LOD}

Clinical features and investigations before LOD were evaluated for every patient. Patients with PCOS were diagnosed according to Rotterdam criteria by menstrual irregularities and ultrasound findings.

56 patients had oligomenorrhea and two cases had regular cycle but, one among them had PCO scan picture and other had a normal scan.

Ultrasound picture of PCOS was present in 56 patients and 2 cases had normal scan, however one patient had oligomenorrhea and one patient had regular cycle.

We looked at the male factor abnormalities associated with sub fertility. Seminal analysis was looked in all 58 cases and it was normal for 50 patients. However, mild male factor abnormality was found in 7 patients and for one patient seminal analysis result was not documented.

\section{Medical treatment before LOD}

We looked at the medical treatment received be-

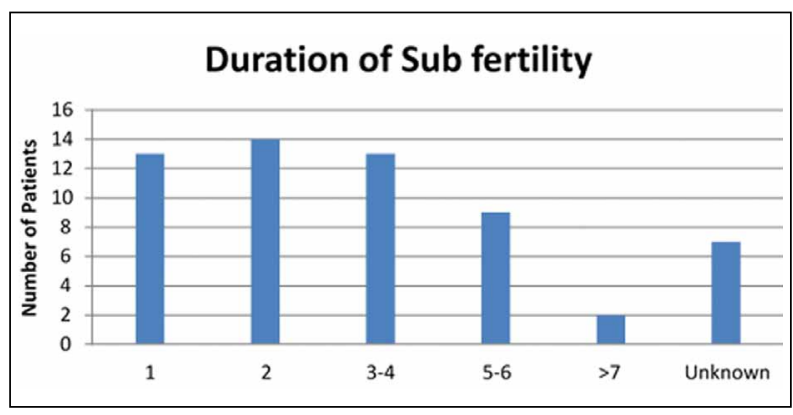

Figure 3. Duration of subfertility before LOD. fore LOD. Most of the patients received clomiphene maximum dose of 50/100mg. However, some received a combination of metformin with clomiphene and only a few received gonadotrophins as ovulation induction before LOD.

52 cases had CC only and 10 cases had clomiphene and metformin. Only 2 cases had gonadotrophins as first line of ovarian induction and no reason was found. 9 cases had more than six cycles of CC.

52 cases had CC $(50 / 100 \mathrm{mg})$ and 9 cases had $>6$ cycles. Among these 9 cases $5 / 9$ cases had 7 cycles, $1 / 9$ had 8 cycles, $1 / 9$ had 11 cycles and $1 / 9$ had 12 cycles. The rest of the cases had different cycles of CC. 10 cases had metformin in addition to CC and 2 cases had ovulation induction with gonadotrophins prior to LOD as shown in figure 4.d

\section{Operative findings in LOD}

Operative findings

For all the 58 patients who underwent LOD the operative finding regarding other associated pathologies and tubal patency was evaluated at laparoscopy prior to ovarian drilling. Eighteen

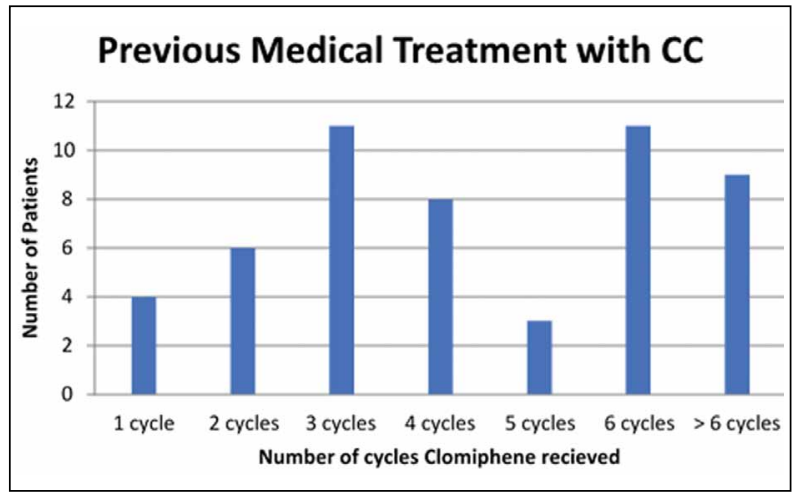

Figure 4. Number of cycles of CC before LOD. 
patients were found to have PCO associated with endometriosis, one patient had endometriosis plus adhesions and seven patients had PCO associated with pelvic inflammatory disease and adhesions. In addition, there were 33 patients with only PCOS and among them one patient had ovarian cyst and other one had rudimentary horn as shown in figure 5 .

\section{Tubal patency}

Tubal patency was checked in all 58 patients at LOD prior to ovarian drilling. In two patients both tubes were blocked, and eleven patients only had one tube blocked. Moreover in 45 patients both tubes were patent as shown in figure 6 .

\section{Operative complications}

There were no records of intra -operative or postoperative complication in all 58 patients. In addition, all the procedures were completed laparoscopically.

\section{Number of Drill Holes}

There were different number of holes made in each ovary, depending on the ovarian size and volume, table 4 shows the number of drill holes in each

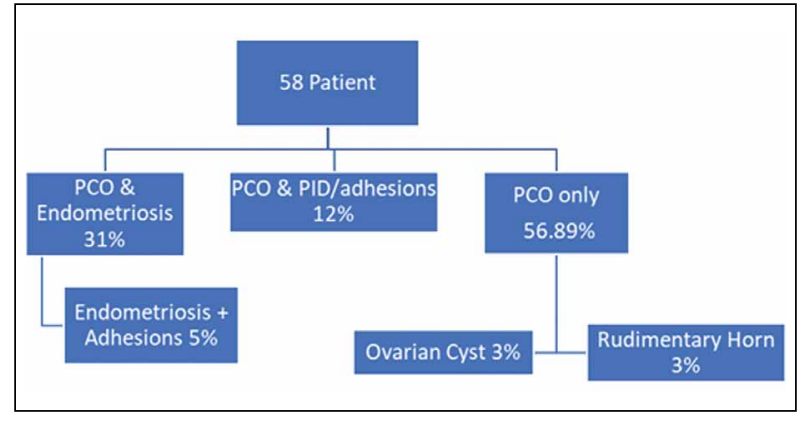

Figure 5. Operative findings in LOD.

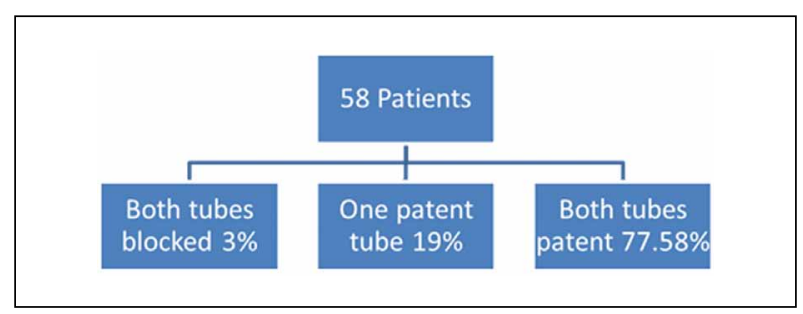

Figure 6. Tubal patency done at LOD.

ovary for the patients. There was no departmental protocol for the number of holes to be made at the LOD so, the decision was based on the presentation of finding at laparoscope.

Table 4. Number of drill holes in the left and right ovaries of 58 patients.

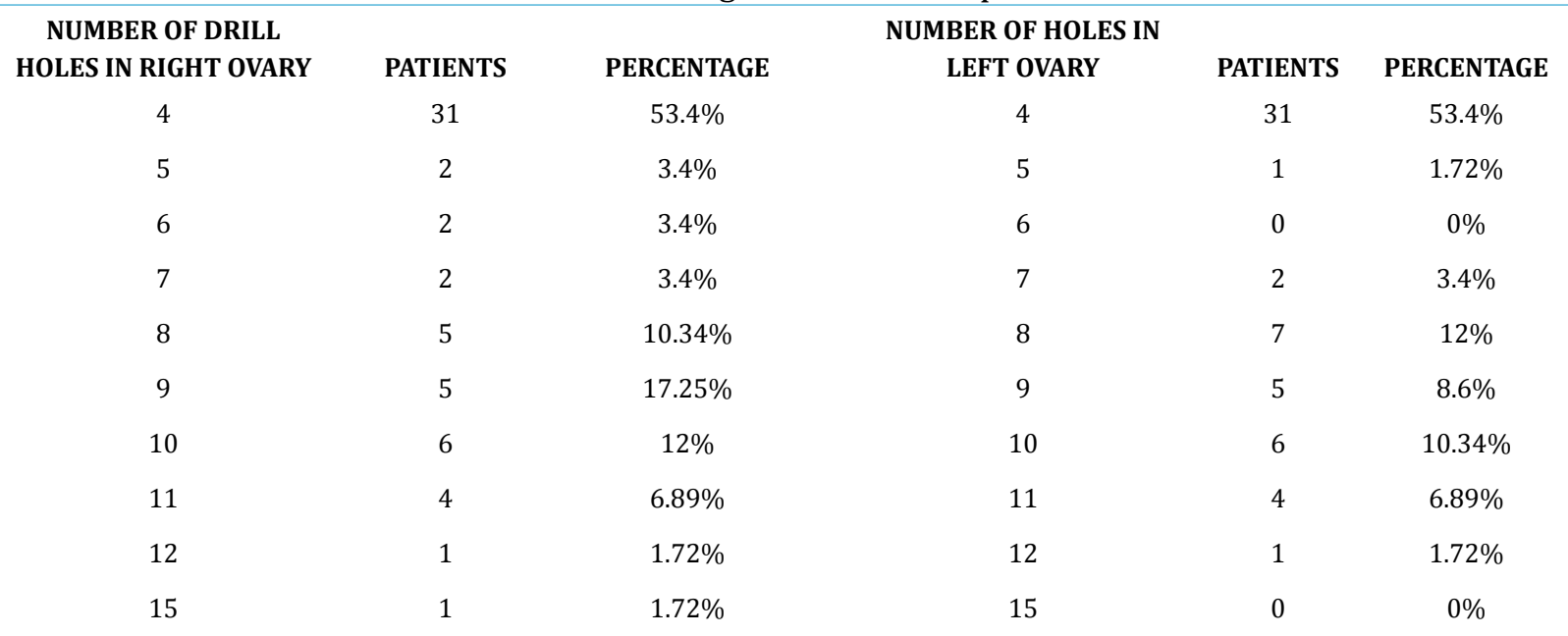




\section{Success Rates}

\section{Ovulatory status post $L O D$}

31 patients had regular ovulation every 4-5weeks after LOD. Moreover, four patients became pregnant before a further follow up appointment. Sixteen patients had irregular cycles after LOD. Cycle length was not documented in five patients and there was no follow found up in two patients. Therefore, a total of 35 patients out of 51 patients got regular ovulation after LOD. Furthermore, comparing the ovulatory status with Cochrane Review (Farquhar et al., 2012 ) it was $68.6 \%$ vs. $71 \%$.

\section{Outcome of LOD}

There was a total of 17 spontaneous pregnancies within one year after LOD, table 5 shows more data on this.

Table 5. Outcome of LOD within a year.

$\begin{array}{cc}\begin{array}{c}\text { DURATION FROM TIME } \\ \text { OF LOD }\end{array} & \text { NUMBER OF SPONTANEOUS } \\ \text { PREGNANCIES } \\ \text { 1-3 months } & 7 \\ 4-6 \text { months } & 2 \\ 7-12 \text { months } & 7 \\ >12 \text { months } & 1\end{array}$

\section{Post LOD}

There were 41 patients who received subsequent treatment. Of the 41 patient who did not conceive after LOD, most of them went into other treatment. 16 patients received CC only after LOD and 6 (39\%) patients were pregnant. In addition, 3 patients received CC and metformin and 2 patients got pregnant. However, 3 patients received metformin only and did not conceive.

Twelve patients had further ovarian induction with FSH, and 3 patients conceived. Moreover, 10 patients underwent IVF and 6 patients became pregnant. This is shown in figure 7.

Looking at the total outcome of pregnancy after LOD with or without further treatment, there was a total number of 34 pregnancies out of 58 cases (58.6\%). There was no significant difference between the two groups when analysing the pregnancy rate. There were 17 (28.3\%) spontaneous pregnancies and $17(28.3 \%)$ pregnancies after further treatment. However, the live birth rate although not statistically relevant showed a weak relationship between two groups. Out of 34 pregnancies there were 29 singleton live births (85.3\%). Those pregnancies that did not result in live birth include 4 first-trimester miscarriages $(11.7 \%)$ and 1 ectopic pregnancy (2.9\%).

\section{Outcome of spontaneous pregnancies in pure PCOS women.}

Looking at the pure PCOS patients found at LOD there were 13 spontaneous pregnancies (39.4\%) and ten had live birth (30.3\%). There were 2 early

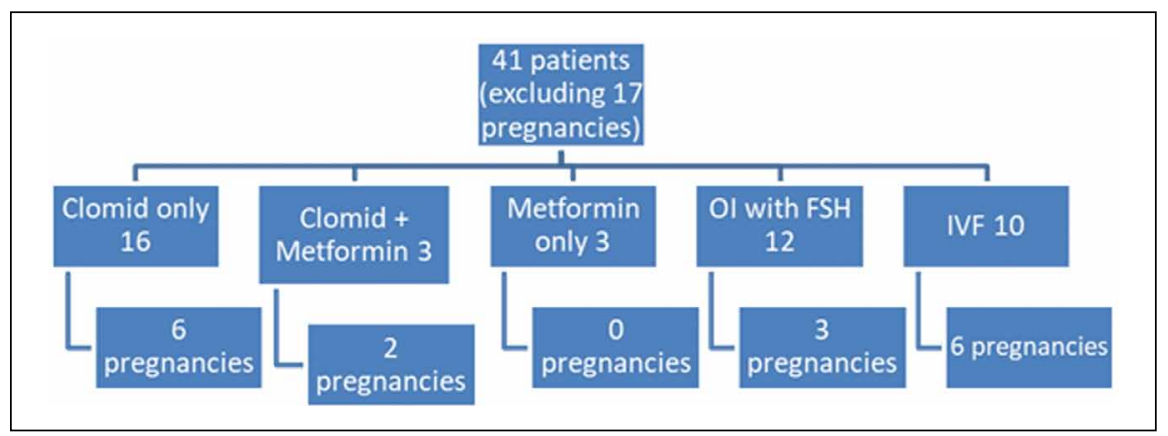

Figure 7. Different consequent treatments underwent by the 41 patients who did not conceive after LOD. 
miscarriages. Moreover, there was one ectopic pregnancy in pure PCOS as shown in figure 8.

\section{Spontaneous pregnancy rates} after LOD and BMI

The impact of body mass index on reproductive outcome after LOD in PCOS. There were 41 patients who had BMI less than 30 and 14 (34.1\%) patients were pregnant in that group. Whereas, there were 9 patients 31-35 BMI and only 1 (11.1\%) patient was found pregnant in that group. There were 4 patients with BMI more than 35 however there was no pregnancy found in that group.

\section{Rate of spontaneous pregnancy vs. associated pathologies}

There were 7 patients with PCO and adhesions; however, there were no spontaneous pregnancies. Furthermore, there were 18 patients found to have PCO with endometriosis, there were 4 spontaneous pregnancies $(22.2 \%)$. There were also 33 patients with pure PCO (only PCO picture at laparoscopy) with a spontaneous pregnancy rate of 13 (39.4\%) as shown in figure 9.

\section{Pregnancy Outcomes after LOD with or without further treatment}

There was a total of 34 pregnancies which is $58.6 \%$ of the 58 cases. Out of these pregnancies there were 17 spontaneous $(28.3 \%)$ and 17 pregnancies were

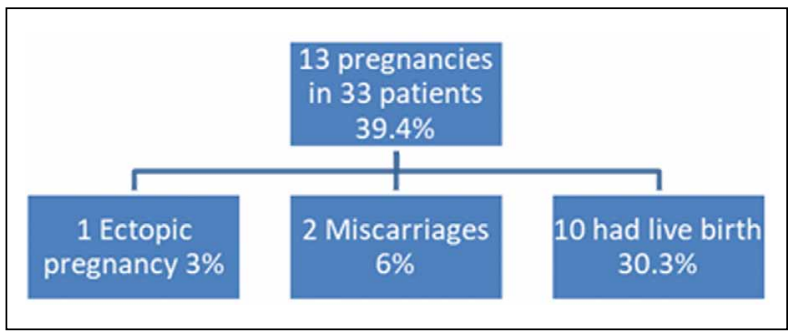

Figure 8. Outcome of spontaneous pregnancies in pure PCOS patients. conceived after further treatment. 29 out of the 34 pregnancies were live births which is $85.3 \%$. However, there were also 4 miscarriages, $11.7 \%$, and 1 ectopic pregnancy, which is $2.9 \%$.

\section{Comparison of LOD results with Cochrane \\ Review in PCOS patients with no pathology:}

As there was no clinical protocol or guidelines for LOD we standardised our audit based on Cochrane review in PCOS without any pathology. For the audit standard the Cochrane database for laparoscopic ovarian drilling by diathermy or laser for ovulation induction in anovulatory PCOS was used. This is a review of a previously updated 9 RCT's and additional 16 trials added to the current update. These studies included PCOS only, i.e. without other pathology. Summary of Cochrane Review of LOD: Ovulation rate was $71 \%$ of women who had undergone bilateral drilling (Six trials including 525 women). The proportion of women who became pregnant ranged from $25 \%$ to $51 \%$ (18 studies reported this outcome in 1930 women). The proportion of live births following LOD ranged from $24 \%$ to $44 \%$ (8 trials including 1034 women) and the proportion of women who suffered miscarriage ranged from 4\% to 9\% (15 trials including 1592 women). The rates of multiple pregnancies

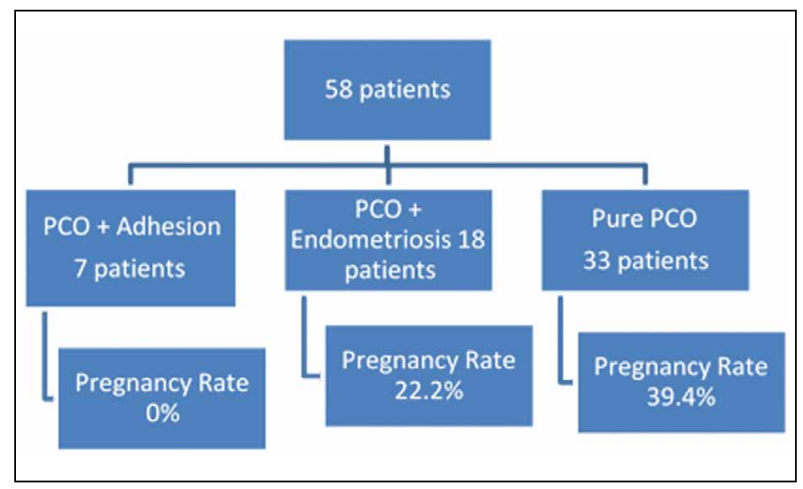

Figure 9. Rate of spontaneous pregnancies in correlation to the associated pathologies. 
were significantly lower in LOD compared to trials using gonadotrophins ( 5 trials including 166 women).

We compared the ovulation rate, pregnancy rate, live birth and miscarriage rate. The difference in the live birth was the greatest statistical finding with median being much lower in our audit (30.3\%).

The rates of ovulation, pregnancy rate, live birth and miscarriage are shown in the table 6. However, the ovulation rate in Cochrane review was $71 \%$ and our local audit showed $68.6 \%$ ovulation. In Cochrane review pregnancy rate was between $25-51 \%$ and our audit showed a pregnancy rate of $39.4 \%$. All live births in Cochrane review were singleton pregnancies and the rate was between $24-44 \%$ and our audit showed $30.3 \%$ live births. Although the miscarriage rate was between 4-9\% in Cochrane review and our audit showed miscarriage rate of $15.4 \%$. There was no record found about the cause of miscarriage and their management. However, comparing this audit to Cochrane review which has included patients with pure PCOS who went LOD has shown a good outcome. Table 6 shows this comparison between our results and those of the Cochrane review.

Table 6. shows the comparison of our LOD results with the Cochrane review for PCOS patients with no other pathology. The table shows the ovulation rate, pregnancy rate, live birth rate and miscarriage rate in PCOS patients who had LOD.

\begin{tabular}{lcc}
\hline & COCHRANE REVIEW & LOCAL AUDIT \\
Ovulation Rate & $71 \%$ & $68.6 \%$ \\
Pregnancy Rate & $25 \%-51 \%$ & $39.4 \%$ \\
Live birth Rate & $24 \%-44 \%$ & $30.3 \%$ \\
Miscarriage Rate & $4 \%-9 \%$ & $15.4 \%$
\end{tabular}

\section{Discussion}

Since the first revised surgical approach to patients of PCOS with LOD in 1984 by Gjonaess, it is evident from the wealth of literature to date that this treatment method remains to be valuable in ascertaining/restoring fertility in women diagnosed with amenorrhea in particularly PCOS.

The aim of the first study was to evaluate the strength of evidence documenting the effectiveness of LOD. Preliminary data has shown that the ovulation rate using LOD has been generally high. Many studies have reported high ovulation rate (80\%) and pregnancy rate of $(60 \%)$. Nonetheless, the pregnancy rate appears not to be remarkably consistent as many clinical factors affect the pregnancy rate. However, the predictors of success of LOD depend on the factors and are important to find before selection of cases for LOD. As per recent studies by Hatem in 2014 the outcome of LOD depends on the body index, serum testosterone concentration, free androgen index and duration of fertility. Recent retrospective study by Fukhara in 2014 showed that logistic regression analysis demonstrated that preoperative FSH values was a significant predictive factor for the success of LOD $p=0.02$; OR 1.8; confidence interval, 1.1-2.9). Similar observational study by Kaur (2013) showed that patients with high value of LH/FSH ratio stay non pregnant despite LOD. There have been many reports on ovulation data and pregnancy rate following LOD in primary and secondary amenorrhoea patients, however Cochrane review has recently reported outcome in patients who only suffer from PCOS. The present study confirms the effectiveness of LOD in both cohorts of patients (pure PCOS or PCO associated with other pathologies), however with a trend to greater ovulation and pregnancy rate in patients with only PCOS.

The retrospective study was carried out to determine the success of LOD in routine practise at East Sussex Health Care NHS Trust. This study provided details of outcome in both patients with pure PCOS and with PCO associated with other pathologies.

\section{Concluding remarks}

Extensive research during the last three decades has shown that women who are clomiphene re- 
sistant can be treated with gonadotrophins but often have an over production of follicles and are exposed to the risks of ovarian hyper stimulation syndrome (OHSS) and multiple pregnancy. Although effective, gonadotrophins are an expensive and time-consuming form of treatment requiring intensive monitoring.

An alternative is surgical therapy using laparoscopic ovarian drilling (LOD) ${ }^{17}$. LOD can also be used for PCOS women who fail to become pregnant despite ovulatory on clomiphene, as a second line treatment. LOD or gonadotrophins can be offered after failure of clomiphene citrate to achieve pregnancy ${ }^{18}$. The mechanism of action of LOD is thought to be like that of ovarian wedge resection. Both procedures may destroy ovarian androgen-producing tissue and reduce the peripheral conversion of androgens to oestrogens. A fall in the serum levels of androgens and LH, and an increase in FSH levels have been demonstrated after ovarian drilling. It restored the hormonal environment to normal by correcting disturbances of the ovarian-pituitary feedback mechanism. Thus, both local and systematic effects are thought to promote follicular recruitment, maturation and subsequent ovulation, hence contributing to its success. 600 patients have been described in the literature that underwent LOD so the data is reliable and has strong evidence that LOD should be offered to certain group of patients with PCOS.

However, the question remains: Is this therapy safe and the first choice of treatment for infertility in clomiphene resistant PCOS patients? Or have the discoveries of fertiloscopy, TVOD, trans-ultrasound ovarian drilling, other invasive techniques and never pharmacotherapy introduced an era of novel management for improving reproduction function? Most of the techniques described above would apply principally to PCOS patients thus LOD remains an adequate model of inducing ovulation in PCOS patients.

\section{Disclosure of Interests}

No conflicting interest on my part, or for any other authors.

\section{Contribution of authorship}

Mr Jamal Zaidi was my internal supervisor and Professor Joyce Harper was my external supervisor. Neither contributed to the writing of this article, however, they have marked it as a part of the requirement from the university.

Mr Zaidi, though he did not physically write any of the manuscript, he was actively involved in the clinical work of the patients whom I audited. It was under his guidance that I was able to complete the project and fully understand the impacts and outcomes of LOD.

Miss Nabeha Dhar strongly contributed by editing, proofreading and formatting both this article and the original thesis.

\section{Details of ethics approval}

No ethics approval was needed

\section{Funding}

I received no funding or grants during the writing process.

\section{References}

1. Achermann, J C et al., 2002. Genetic Causes of Human Reproductive Disease. The Journal of Clinical Endocrinology \& Metabolism 87(6), 2447-2454.

2. Farah, L et al., 1999. Prevalence of polycystic ovary syndrome in women seeking treatment from community electrologists. Alabama Professional Electrology Association Study Group. The Journal of Reproductive Medicine. 44(10) 870-4.

3. Diamanti-Kandarakis, E et al., 2006. The role of genes and environmental in the etiology of PCOS. Endocrine. Human Reproduction (Oxford, England). 21(6), 1426-31. 
4. Zumoff, B et al., 1983. A Chronobiologic Abnormality in Luteinizing Hormone Secretion in Teenage Girls with the Polycystic-Ovary Syndrome. The New England Journal of Medicine. 309(20), 1206-9.

5. Legro, R S et al., 1998. Polycystic ovarian syndrome: Current and future treatment paradigms. American Journal of Obstetrics and Gynaecology. 179(6), S101-8.

6. Bremer, A A et al., 2010. Polycystic Ovary Syndrome in the Pediatric Population. Metabolic Syndrome And Related Disorders, University of Chicago. 8(5), 375-93.

7. Guzick, D S et al., 2004. Polycystic Ovary Syndrome. The American College of Obstetricians and Gynaecologists. 103(1), 181-93.

8. Tang, T et al., 2012. Insulin-sensitising drugs (metformin, rosiglitazone, pioglitazone, D-chiro-inositol) for women with polycystic ovary syndrome, oligo amenorrhoea and subfertility. Cochrane Database System. Rev. 5, CD003053.

9. Farquhar, C et al., 2012. Laparoscopic drilling by diathermy or laser for ovulation induction in anovulatory polycystic ovary syndrome. The Cochrane Library. Issue 6. Art. No.: CD001122. DOI: 10.1002/14651858.CD001122.

10. Armar, N A et al., 1990. Laparoscopic ovarian diathermy in the management of anovulatory infertility in women with polycystic ovaries: endocrine changes and clinical outcome. 53(1), 45-9.

11. Keckstein, J et al., 1989. Laparoscopic treatment of polycystic ovarian syndrome. Bailliere's Clinical Obstetrics and Gynaecology. 3(3), 563-81

12. Balen, A H et al., 1993. Hypersecretion of luteiniz- ing hormone and the polycystic ovary syndrome. Human Reproduction (Oxford, England). 8 Suppl 2, 123-8.

13. Amer, Saka et al., 2002. Long-term follow-up of patients with polycystic ovary syndrome after laparoscopic ovarian drilling: endocrine and ultrasonographic outcomes. Human Reproduction (Oxford, England). 17(11), 2851-7.

14. Zahiri Sorouri, Z et al., 2015. Comparison between Unilateral and Bilateral Ovarian Drilling in Clomiphene Citrate Resistance Polycystic Ovary Syndrome Patients: A Randomized Clinical Trial of Efficacy. International Journal of Fertility \& Sterility. 9(1), 9-16.

15. Samy N et al., 2014 Serum Vascular Endothelial Growth Factor in Polycystic Ovary Syndrome and its Relation to Ovarian Drilling. International Journal of Toxicological and Pharmacological Research. 6(4), Pp123-7.

16. Ezenwa $C$ et al., 2012. Laparoscopic ovarian drilling for polycystic ovary syndrome (PCOS) - are we wasting women's time. International Journal of Obstetrics \& Gynaecology. 119, 140-1.

17. Gjoannaess H. Polycystic ovarian syndrome treated by the electrocautery through the ovarian laparoscope. Fertil Steril. 1984;41:20-5.

18. Qiao, J., Yang, Y. \& Li, R. Polycystic Ovary Syndrome: Fertility Management. Curr Obstet Gynecol Rep 1, 159-165 (2012). https://doi.org/10.1007/ s13669-012-0027-2.

Received 30-08-21

Revised 06-09-21

Accepted 20-09-21 